\title{
Soft gamma-ray Galactic ridge emission as unveiled by SPI aboard INTEGRAL
}

\author{
J. Knödlseder*, V. Lonjou ${ }^{\dagger}$, G. Weidenspointner*, P. Jean*, A. Strong ${ }^{* *}$, R. Diehl ${ }^{* *}$, \\ B. Cordier ${ }^{\ddagger}$, S. Schanne ${ }^{\ddagger}$ and C. Winkler ${ }^{\S}$ \\ ${ }^{*}$ CESR (UPS/CNRS), 9, avenue du Colonel Roche, BP 44346, 31028 Toulouse Cedex 5, France \\ ${ }^{\dagger}$ CENBG, Université Bordeaux 1 (CNRS/IN2P3), Chemin du Solarium, BP 120, 33175 Gradignan, France \\ ${ }^{* *}$ Max-Planck-Institut für extraterrestrische Physik, Giessenbachstrağe, 85748 Garching, Germany \\ ${ }^{\ddagger}$ CEA Saclay, DSM/DAPNIA/Service d'Astrophysique, 91191 Gif-sur-Yvette, France \\ ${ }^{\S}$ ESA/ESTEC, SCI-SA Astrophysics Division, 2201 AZ Noordwijk, The Netherlands
}

\begin{abstract}
The origin of the soft gamma-ray $(200 \mathrm{keV}-1 \mathrm{MeV})$ Galactic ridge emission is one of the long-standing mysteries in the field of high-energy astrophysics. Population studies at lower energies have shown that emission from accreting compact objects gradually recedes in this domain, leaving place to another source of gamma-ray emission that is characterised by a hard power-law spectrum extending from $100 \mathrm{keV}$ up to $100 \mathrm{MeV}$. The nature of this hard component has remained so far elusive, partly due to the lack of sufficiently sensitive imaging telescopes that would be able to unveil the spatial distribution of the emission. The SPI telescope aboard INTEGRAL allows now for the first time the simultaneous imaging of diffuse and point-like emission in the soft gamma-ray regime. We present here all-sky images of the soft gamma-ray continuum emission that clearly reveal the morphology of the different emission components. We discuss the implications of our results on the nature of underlying emission processes and we put our results in perspective of GLAST studies of diffuse Galactic continuum emission.
\end{abstract}

Keywords: Gamma-rays; Gamma-ray sources; Cosmic Rays

PACS: $95.85 . \mathrm{Pw} ;$ 98.70.Rz; 98.70.Sa

\section{INTRODUCTION}

The lack of sensitive imaging telescopes covering the soft gamma-ray (200 keV $-1 \mathrm{MeV})$ band has so far prevented the study of Galactic emission processes in this energy band. Surveys at lower energies, such as those performed by HEAO-1 and RXTE, indicate that the Galaxy is dominated by strong point-source emission from X-ray binary systems. In addition, a diffuse emission component is observed below $\sim 60 \mathrm{keV}$ that may eventually be attributed to unresolved emission from a large number of CV systems [5]. Surveys at higher energies, such as those performed by the COMPTEL and EGRET telescopes aboard CGRO, reveal primarily diffuse emission from the Galactic plane. While above $\sim 100 \mathrm{MeV}$ the emission is relatively well understood as the result of cosmic-ray interactions with the interstellar medium, the observed $1-100 \mathrm{MeV}$ emission is in excess of predictions from cosmic-ray propagation models [6].

The transition region around $200 \mathrm{keV}-1 \mathrm{MeV}$, where diffuse emission components should start to dominate pointsource emission, has so far never been imaged. The imaging spectrometer SPI aboard INTEGRAL combines for the first time good sensitivity and imaging performances to allow mapping the sky in this energy domain. First attempts to detect the diffuse emission component with SPI using model fitting techniques have been reported by [7] and [1]. The results clearly indicate the presence of a diffuse emission component that starts to dominate over the point-source emission above 100-200 keV.

In this paper we report about work in progress that aims in direct imaging of this diffuse emission. The imaging is done using a multiresolution deconvolution procedure called MREM [3, 4]. The data that were analysed in this work consist of those included in the December 8, 2006 public INTEGRAL data release. They cover the period IJD 1074 - 2120, spanning almost 3 years of INTEGRAL mission data. With this dataset, $85 \%$ of the sky is covered with an exposure above $10 \mathrm{ks}$, corresponding to a INTEGRAL/SPI point-source sensitivity of $\sim 160 \mathrm{mCrab}$ in the $150-300$ $\mathrm{keV}$ band. Most of the Galactic plane has an exposure in excess of $1 \mathrm{Ms}$, corresponding to a INTEGRAL/SPI pointsource sensitivity of $\sim 16 \mathrm{mCrab}$ in the $150-300 \mathrm{keV}$ band. 


\section{SKYMAPS}

Skymaps have been generated from INTEGRAL/SPI data using the MREM multiresolution deconvolution algorithm [3, 4] for a number of energy bands. Due to the space limitations we show in Fig. 1 only the maps obtained for the $150-300 \mathrm{keV}$ and $300-500 \mathrm{keV}$ energy bands. A full set of maps can be seen at http://integral.esac.esa.int/POMNov2006.html
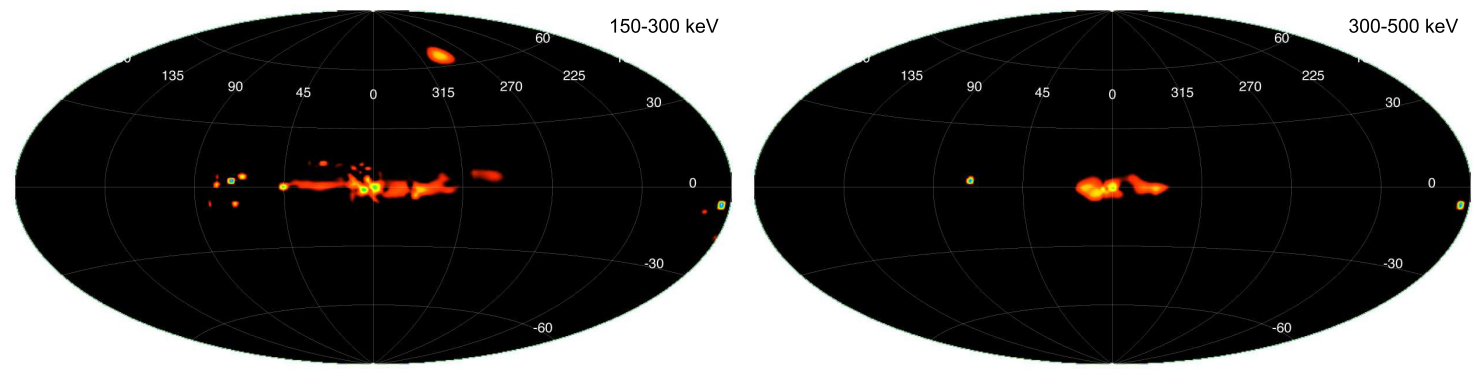

FIGURE 1. INTEGRAL/SPI multiresolution maps in the energy bands 150-300 keV (left) and 300-500 keV (right).

Figure 1 clearly reveals the presence of diffuse emission in both energy bands. While in the $150-300 \mathrm{keV}$ band still a number of point-sources are seen, they gradually fade away at higher energies. Note that diffuse emission in this context does not necessarily mean that the underlying emission mechanism is indeed diffuse. The apparent morphology may also be explained by a number of faint and unresolved point sources.

To reveal better the diffuse Galactic ridge emission, we remove known point-sources from the image by fitting their contribution in the data-space while deconvolving the residual counts into an allsky image. The flux of each source in the catalogue is adjusted together with the level of the instrumental background during the image reconstruction procedure. The multiresolution algorithm picks up any residual structure in the data that is not fit by the model components. Some cross talk exists between diffuse emission and point-source emission due to the limited angular resolution of SPI (FWHM of $\sim 3^{\circ}$ ) and the low detection significance for many of the sources. Also numerous faint sources that are below the SPI detection threshold can not be resolved by the method. Consequently, the residual emission seen in these point-source subtracted skymaps may be either explained by truly diffuse emission, the combined emission of weak and unresolved point sources, or by residual point source emission.
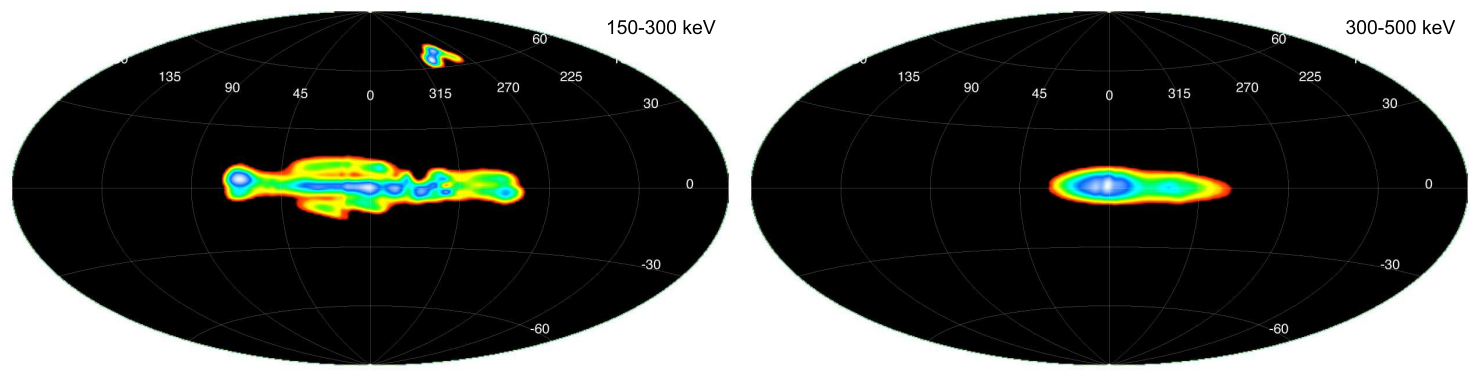

FIGURE 2. Point-source subtracted INTEGRAL/SPI multiresolution maps in the energy bands 150-300 keV (left) and $300-500$ $\mathrm{keV}$ (right).

Figure 2 shows the point-source subtracted maps for the energy bands $150-300 \mathrm{keV}$ and $300-500 \mathrm{keV}$. In the $150-$ $300 \mathrm{keV}$ band an extended and narrow Galactic ridge emission is seen, reaching from the Cygnus region (at longitude $\sim 80^{\circ}$ ) to the Carina region (at longitude $\sim 280^{\circ}$ ). Some of the structure seen in the map may be related to source variability, that has explicitly only be taken into account for the strongest known point-sources sources. We therefore caution not to over interpret morphology details, and we are currently working on an improved treatment of source variability in the analysis.

In the 300-500 keV band the emission appears much more concentrated towards the inner Galaxy. This concentration can be understood from the increasing contribution of Positronium continuum emission which amounts to roughly half of the Galactic emission seen in the image. As we know from dedicated analyses, the positronium continuum morphology can be well described by a symmetrical Gaussian shaped bulge with FWHM of $\sim 8^{\circ}$ located at the 
Galactic centre [9]. Whether the apparent asymmetry of the underlying Galactic ridge emission is linked to positronium continuum emission or Galactic continuum emission remains to be examined by a dedicated spectral analysis.

\section{CONCLUSIONS}

We have presented allsky maps of continuum emission in the $150-300 \mathrm{keV}$ and $300-500 \mathrm{keV}$ energy bands that clearly show the presence of a diffuse or unresolved Galactic emission component. Known diffuse emission mechanism, such as inverse Compton and bremsstrahlung processes, seem insufficient to explain the observed intensity levels (Strong et al., these proceedings). In-situ electron acceleration could be a possible way to produce the observed emission without violating any energetic constraints [2]. In-flight annihilation of high-energy positrons or cosmic-ray knock-on secondary electrons could also contribute to the emission.

Alternatively, the apparently diffuse emission could arise from an unresolved population of weak sources. Population synthesis indicates that a source population with a hard spectral index could indeed produce the observed emission without violating any source counts constraint [8]. If such a source class indeed exists it may also have implications on diffuse emission studies in the GLAST energy range. Conversely, GLAST may even help to unveil the source population. If, on the other hand, the soft gamma-ray Galactic ridge emission turns out to be intrinsically diffuse, the required modification of the cosmic-ray propagation models will necessarily also impact the analysis in the GLAST domain. Building a coherent image of Galactic ridge high-energy emission will therefore be a major task within the next decade.

\section{ACKNOWLEDGMENTS}

The SPI project has been completed under the responsibility and leadership of CNES. We are grateful to ASI, CEA, CNES, DLR, ESA, INTA, NASA and OSTC for support.

\section{REFERENCES}

1. L. Bouchet, J.-P. Roques, P. Mandrou, et al., ApJ, 635, 1103 (2005).

2. V. A. Dogiel, I. Hajime, K. Masai, et al., ApJ, 581, 1061 (2002).

3. J. Knödlseder, D. Dixon, K. Bennett, et al., A\&A, 345, 813 (1999).

4. J. Knödlseder, G. Weidenspointner, P. Jean, et al., "Imaging the Gamma-Ray sky with SPI aboard INTEGRAL," in The 6th INTEGRAL Workshop, edited by S. Grebenev, R. Sunyaev and C. Winkler, ESA SP-622, 2007, in press.

5. R. Krivonos, M. Revnivtsev, E. Churazov, et al.,A\&A, 463, 957 (2007).

6. A. W. Strong, I. V. Moskalenko, and O. Reimer, ApJ, 537, 763 (2000).

7. A. W. Strong, R. Diehl, H. Halloin, et al., $A \& A$, 444, 495 (2005).

8. A. W. Strong, "Source population synthesis and the Galactic diffuse gamma-ray emission," in The multi-messenger approach to high-energy gamma-ray sources, Astrophysics and Space Science, in press.

9. G. Weidenspointner, C. R. Shrader, J. Knödlseder, et al., A\&A, 450, 1013 (2006). 